\title{
Modeling and Forecast of Ghana's GDP Using ARIMA-GARCH Model
}

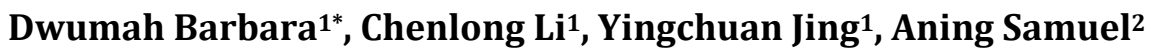 \\ ${ }^{1}$ Department of Statistics, Taiyuan University of Technology, Taiyuan, China \\ ${ }^{2}$ Institute of Computer Science, University of Silesia in Katowice, Katowice, Poland \\ Email: ^dwumahbarbie@gmail.com, lichenlong@tyut.edu.cn, shyjyc1970@163.com, samuel.aning@us.edu.pl
}

How to cite this paper: Barbara, D., Li, C.L., Jing, Y.C. and Samuel, A. (2022) Modeling and Forecast of Ghana's GDP Using ARIMAGARCH Model. Open Access Library Journal, 9: e8335.

https://doi.org/10.4236/oalib.1108335

Received: December 28, 2021

Accepted: January 26, 2022

Published: January 29, 2022

Copyright $\odot 2022$ by author(s) and Open Access Library Inc.

This work is licensed under the Creative Commons Attribution International License (CC BY 4.0).

http://creativecommons.org/licenses/by/4.0/

(c) (i) Open Access

\begin{abstract}
GDP is frequently used as a way of national evaluations, as well as a way of measuring economic progress. This paper analyses a combination of time series models that are both linear and non-linear in making forecast of Ghana's GDP. Ghana's GDP current prices data from 1980 to 2019 were used in the analysis. Based on the AIC values, the best model was determined to be ARIMA $(2,2,2)$ in modeling our data, except that it is heteroscedastic. The combination with non-linear GARCH $(1,1)$ model is used to capture these variances over time. The diagnostics test further shows that the presented model is stable and quite reliable. The results of the study reveal that the GDP of Ghana will continue to increase for the next 10 years and this goes to show that the nation is moving forward.
\end{abstract}

\section{Subject Areas}

Mathematical Statistics

\section{Keywords}

Gross Domestic Product (GDP), Heteroscedasticity, ARIMA, GARCH, Volatility

\section{Introduction}

GDP is frequently used as a way of national evaluations, as well as a way of measuring economic progress. It is widely regarded as the most effective statistical indicator of national development and progress in the world [1]. The term "Gross Domestic Product" (GDP) refers to the total monetary worth of all final goods and services produced (and sold on the market) within a country over a given timeframe [2]. GDP also forms a major part when calculating the human 
development index of a particular country, which is used as yardstick for measuring the development of a country [3]. If GDP is increasing, the state of the economy is strong and the nation is progressing. Alternatively, if GDP declines, the economy could be in difficulty, and the country could lose progress [4]. Since Ghana in 2019 was predicted to have the fastest growing economy in the world, with words like "skyrocketing" being used to describe its expansion [5] [6], the future direction of Ghana's economic growth has become a hotly debated topic among legislators and researchers alike.

As a result of the extensive practical significance of forecasting, a broad diversity of various forecasting methods has gradually come into existence, with time series models being widely used especially for GDP [7]. ARIMA models and exponential smoothing methods are two well-known examples of methods for predicting specific time series [8]. Using the Box-Jenkins method, Asenso et al. (2017) forecasted Ghana's GDP for the period 2015-2020 using the ARIMA (0, 1, 0 ) model. Their study was based on GDP data from 1970 to 2014 and highlighted that foreign factors have a significant impact on Ghana's GDP [9]. Zhou et al. (2014) used ARIMA/GARCH method in modeling and predicting network traffic. They showed that the model could capture prominent traffic characteristics such as long-range dependence (LRD) and self-similarity, not only in large time scale but also in small time scale. They also concluded that the model had better prediction accuracy when compared with existing FARIMA model [10]. Another significant prediction research work was introduced by Higgins et al. (2016) in the case of China. They proposed a benchmark model to make forecast of China's macroeconomy, using Bayesian vector autoregression (BVAR) approach, particularly growth in GDP and inflation in the consumer price index (CPI), which demonstrates the ability to predict turning moments and can be used to analyze policy in a variety of scenarios, allowing density forecasts to be created as well as point forecasts. They concluded that when the root mean square errors are taken into account, their model outperforms dozens of competing models, particularly over long-run forecast horizons [11]. As in [10], Tran et al. (2015) also applied a multiplicative seasonal ARIMA/GARCH model in predicting the traffic of mobile communication network operation in Vietnam. According to them, the model can be considered as flexible in capturing well the characteristics of EVN traffic series and give reasonable forecasting results. Also, they showed in situations that the volatility is not necessary to be taken into account, i.e. short-term prediction, the multiplicative seasonal ARIMA/GARCH model still acts well with the GARCH parameters adjusted to $(0,0)$ [12]. Using lagged GDP growth from other African countries, Eersel (2019) forecasted Ghana's GDP growth using a hybrid of factor-methods and machine learning shrinkage methods. They concluded that using lags from different countries as predictors can aid in the development of a good forecasting model, and the hybrid model predicts Ghana's GDP growth more accurately [13]. In the domain of demand forecasting, one is frequently confronted with 
data that is sporadic, intermittent, or bursty in nature which breach primary assumptions of many traditional techniques such as Gaussian errors, time series stationarity, or homoscedasticity [7].

In this paper, we use the ARIMA-GARCH model to forecast Ghana's GDP. The Autoregressive Integrated Moving Average (ARIMA) with Generalized Autoregressive Conditional Heteroscedasticity (GARCH) model is simply a combination of the linear ARIMA and the non-linear GARCH timeseries. ARIMA models are popular forecasting methods with numerous applications in finance. For example, future returns can be predicted using a linear combination of past returns and residuals. Unfortunately, when the variance of returns changes over time, this family of models encounters difficulties. This is a fairly common occurrence known as conditional variance or volatility clustering. Fortunately, another group of models, the ARCH and GARCH models, can model this irregularity. In practice, both types of models are thus combined to optimize forecasting performance [14].

Hence, now that we know this combination can be used to model heteroscedasticity and improve forecast performance, we are interested in applying this technique to forecast Ghana's GDP. We would also want to know if there is indeed the issue of conditional variance/heteroscedasticity after using only ARIMA model and if the implementation of the GARCH model will indeed take care of this irregularity and improve our forecasts.

To accomplish this, we go through the steps involved in fitting an ARIMA model, followed by fitting a GARCH model to the residuals. This allows the capturing of irregularities in terms of Heteroscedasticity over time. Next, we will apply our models to predict Ghana's GDP for the period 2020 to 2029. We will also compare our forecast values to that of the International Monetary Fund (IMF).

\section{Methodology}

The data used in this paper, is a secondary data acquired from the World Bank. The analysis was carried out using 40 annual observations of the GDP (current US\$) data for the period 1980 to 2019.

\subsection{The ARIMA Model}

The steps involved in fitting the ARIMA model in this paper were taken from [15] and [9]. The Box-Jenkins model is based on two assumptions of the data: stationarity of the autoregressive (AR) model and invertibility of the moving average (MA).

\subsubsection{Stationarity of the AR Models}

The parameters of an AR $(p)$ process $\phi_{1}, \phi_{2}, \cdots, \phi_{p}$, that is, an order $p$ Autoregressive model is given as

$$
\tilde{\mathrm{z}}_{t}=\phi_{1} \tilde{\mathrm{z}}_{t-1}+\cdots+\phi_{p} \tilde{\mathrm{z}}_{t-p}+a_{t}
$$

or 


$$
\left(1-\phi_{1} B-\cdots-\phi_{p} B^{p}\right) \tilde{z}_{t}=\phi(B) \tilde{z}_{t}=a_{t}
$$

For the process to be stationary, certain conditions must be met. For illustration, consider the AR (1) process

$$
\left(1-\phi_{1} B\right) \tilde{z}_{t}=a_{t}
$$

could be written as

$$
\tilde{z}_{t}=\left(1-\phi_{1} B\right)^{-1} a_{t}=\sum_{j=0}^{\infty} \phi_{1}^{j} a_{t-j}
$$

given that the right-hand infinite series converges in a meaningful way. Hence,

$$
\psi(B)=\left(1-\phi_{1} B\right)^{-1}=\sum_{j=0}^{\infty} \phi_{1}^{j} B^{j}
$$

(B) should converge for $|B| \leq 1$ in order for stationarity, or equivalently that $\sum_{j=0}^{\infty}\left|\phi_{1}\right|<\infty$. This implies for an AR (1) process, that the parameter $\phi_{1}$ has to fulfill the condition $\left|\phi_{1}\right|<1$ to guarantee stationarity. Since the root of $1-\phi_{1} B=0$ is $B=\phi_{1}^{-1}$, to put it another way, this condition is the same as declaring that the root of $1-\phi_{1} B=0$ must lie outside the unit circle.

The overall $\operatorname{AR}(p)$ process $\phi(B) \tilde{z}_{t}=a_{t}$ can be written as

$$
\tilde{z}_{t}=\phi^{-1}(B) a_{t}=\psi(B) a_{t}=\sum_{j=0}^{\infty} \psi_{j} a_{t-j}
$$

assuming that the expression on the right-side is convergent. Using the factorization

$$
\phi(B)=\left(1-G_{1} B\right)\left(1-G_{2} B\right) \cdots\left(1-G_{p} B\right)
$$

where the roots of $(B)=0$ are $G_{1}^{-1}, \cdots, G_{p}^{-1}$ and by expanding $\theta^{-1}(B)$ in partial fractions yield

$$
\tilde{z}_{t}=\phi^{-1}(B) a_{t}=\sum_{i=1}^{p} \frac{K_{i}}{1-G_{i} B} a_{t}
$$

As a result, if for $|B| \leq 1, \psi(B)=\phi^{-1}(B)$ is to be a convergent series, meaning, if the weights $\psi_{j}=\sum_{i}^{p} K_{i} G_{i}^{j}$ are going to be entirely summable for the AR ( $p$ ) process to be stationary, then we must have $\left|G_{i}\right|<1$, for $i=1, \cdots, p$. Alternatively, the roots of the $\phi(B)=0$ must lie outside the unit circle. The roots of the equation $\phi(B)=0$ may be known as the zeros of the polynomial $\phi(B)$. Hence, the zeros of $\phi(B)$ should lie outside of the unit circle, in order for stationarity to be reached.

\subsubsection{Invertibility of MA}

A time series $\left\{\tilde{z}_{t}\right\}$ can be said to follow a $q$ order moving average process which is denoted by MA $(q)$ if it can be expressed as

$$
\tilde{z}_{t}=a_{t}+\theta_{1} a_{t-1}+\theta_{2} a_{t-2}+\cdots+\theta_{q} a_{t-q}
$$

where $q$ is the lag in the moving average, $\left(\theta_{1}, \theta_{2}, \cdots, \theta_{q}\right) ; \theta \neq 0$ are the constants and $a_{t}$ is believed to be white noise with zero mean and variance $\delta$. The MA process is always stationary for any value of $\left(\theta_{1}, \theta_{2}, \cdots, \theta_{q}\right)$. Using the back shift 
operator, it is possible to write equation (8) as $\tilde{z}_{t}=\theta(B) a_{t}$ where $\theta(B)=1+\theta_{1} B+\theta_{2} B^{2}+\cdots+\theta_{q} B^{q}$ is the moving average operator or polynomial.

We derive the invertibility condition for the MA $(p)$ model by going through the same steps as that of the $\operatorname{AR}(p)$. This gives us the invertibility condition as: MA $(q)$ model is said to be invertible if and only if $\left|\theta_{j}\right|<1$ for all $j$.

A process $\tilde{z}_{t}$ is considered to be an autoregressive integrated moving average (ARIMA $(p, d, q))$ process if

$$
\Delta^{d} \tilde{z}_{t}=(1-B)^{d} \tilde{z}_{t}
$$

is ARMA $(p, q)$ process. $d$ is a non-negative integer representing the differencing order.

It is possible to write the ARIMA model using the back shift operator as $\phi(B)(1-B)^{d} \tilde{z}_{t}=\theta(B) a_{t}$.

We go through the four major steps which involves model identification, parameter estimation, diagnostic checking and lastly, model use (or forecasting).

\subsection{Model Identification}

The rough methods used on a set of data in order to suggest the type of model that should be investigated further are called Identification methods. The main goal here is to gain certain information of the values of $p, d$, and $q$ required in the overall linear ARIMA model, as well as gain preliminary parameter estimates. This stage entails testing the data for stationarity and seasonality, as well as determining the order of the model's $p, d$, and $q$ components. We used the $\mathrm{ACF}$ and PACF plots, as well as the unit root test known as the Augmented Dickey-fuller (ADF) test, in testing for stationarity. The ADF test employs the unit root test to determine whether or not differencing is required. The fitted ARIMA model is given as

$$
\nabla \tilde{z}_{t}=\mu+\sum_{i=1}^{p} \phi_{i} \tilde{z}_{t-i}+\sum_{j=1}^{q} \theta_{j} a_{t-j}+a_{t}, a_{t} \sim W N\left(0, \delta^{2}\right)
$$

The test hypothesis of the ADF is that

$\mathrm{H}_{0}$ : assumes the presence of unit root i.e., the model is not stationary.

$\mathrm{H}_{1}$ : the model is stationary.

If the data is not stationary, it must be differenced before the integrated (I (d)) component of the Box-Jenkins model can be introduced. If there are some spikes (at regular intervals) in the time plot, the model has a seasonal component. We need to look at the ACF plot to see if there is a seasonal component to the data. If there exist some spikes at regular intervals or lags, then the data contains a seasonal component. We can fix this by taking a moving average of one on the raw data.

\subsection{Estimation of Parameters}

This step has to do with estimating the parameters identified in the ARIMA ( $p$, $d, q)$ model. We can do this by studying the sample autocorrelation function (SACF) and the sample partial autocorrelation function (SPACF) correlogram. 
That is, the correlogram of the differenced data in the case of non-stationarity. Whenever $p=0$ and $q>0$, the SACF shuts off after lag $q$ and the PACF tails off, indicating the order of the MA component. An MA term is technically the lag forecast error. Therefore, the series might be over differenced, if the SACF cuts off after lag $q$ or the SACF at lag 1 is negative. We can correct this by introducing an additional MA component and the significant number of lags in the SACF suggests the $q$-order (which controls the effect of past residuals on the current value).

Furthermore, whenever $p>0$ and $q=0$, the SPACF shuts off after lag $p$ whereas the SACF tails off. As a result, if the SPACF shuts off after lag $p$ or the SACF at lag 1 is positive, then the series may be under differenced. It can be corrected by introducing an additional AR component and the significant number of lags in the SPACF specifies the order of $p$ (which controls the effect of past values on present value). We can rectify any autocorrelation in a stationarized series by adding enough AR terms. Although this method gives a fair idea on the possible order for the ARIMA $(p, d, q)$ model, the best order is selected by checking for the model with the least AIC, AICc or BIC value.

\subsection{Diagnostic Testing of the Model}

After identifying the model and estimating the parameters, we apply diagnostic checks to the fitted model to see how good it is.

The Box-Jenkins model diagnostics are based on the assumption that a good model should have a stationary error term that follows a white noise process. When these requirements are met, the model can be used to make predictions. If not, we fit another model. The residual time plot is used to test for stationarity. The test for the independence and identical distribution (white noise) is done by studying the behavior of the SACF and SPACF of the residuals and the Ljung-Box Test. The Ljung-Box test is given as: $Q^{\star}=(T+2) \sum(T-k) h-1 k=1$ $\rho k 2$, where $h$ is the maximum lag under consideration, $T$ denotes the number of observations and $k$ is the number of model parameters. The test is to check if the first $h$ autocorrelation coefficients differ from that which is expected from the error terms. A high $Q$ value indicates that the correlation did not come from a white noise series. The assumption here is that, if $Q^{*}$ follows the Chi-square distribution, then correlation was obtained from a white noise process. That is $Q \sim \chi^{2}$ with $(h-k)$ degrees of freedom.

The hypothesis is given as follows: $\mathrm{H}_{0}$ : The error terms are i.d.d random variables, and $\mathrm{H}_{1}$ : The error terms are not i.d.d random variables. If we realize that the model does not follow the white noise process, then a new model is required instead of making forecast with the old one. The normal Q-Q plot is used to test the white noise component and the normality assumption.

\subsection{The GARCH Model}

Reference [16] describes a development of the ARCH model that integrates an 
MA component together with the AR component as a Generalized Autoregressive Conditional Heteroskedasticity (GARCH). According to [17], a time series $\left\{\epsilon_{t}\right\}$ is given at each instance by:

$$
\epsilon_{t}=\sigma_{t} w_{t}
$$

where $\left\{w_{t}\right\}$ is discrete white noise, with zero mean and unit variance, and $\sigma_{t}^{2}$ is given by:

$$
\sigma_{t}^{2}=\alpha_{0}+\sum_{i=1}^{q} \alpha_{i} \in_{t-i}^{2}+\sum_{j=1}^{p} \beta_{j} \sigma_{t-j}^{2}
$$

where $\alpha_{i}$ and $\beta_{j}$ are the model parameters.

We say that $\left\{\epsilon_{t}\right\}$ is a GARCH model of order $p, q$ (where the order of the GARCH terms $\sigma^{2}$ is $p$ and $q$ is the order of the ARCH terms $\epsilon^{2}$ ).

Hence from [15], combining the two models gives the ARIMA-GARCH model expressed as

$$
\tilde{z}_{t}=\mu+\sum_{i=1}^{p} \phi_{i} \tilde{z}_{t-i}+\sum_{j=1}^{q} \theta_{j} \epsilon_{t-j}+\epsilon_{t}, \quad \epsilon_{t} \sim W N\left(0, \delta^{2}\right)
$$

where $\epsilon_{t}$ and $\sigma_{t}^{2}$ are given above.

Here, the GARCH model is fitted on the residuals of the ARIMA model instead of the GDP. The initial $p$ and $q$ GARCH parameters should be separately estimated and compared to the ARIMA parameters. The autocorrelation function and partial autocorrelation function can also be used in determining the order of the GARCH parameters, however, in most cases, these orders are not quite precise [10]. According to [18] [19], it is adequate for capturing the performance of the variance by setting the order of $p$ and $q$ to 1 .

\section{Implementation of the Arima-Garch Method on the Data}

\subsection{Model Identification}

The time plot in Figure 1 shows our time series data is not stationary and shows an upward trend. Figure 2 and Figure 3 show the ACF and PACF plots. The $\mathrm{p}$-value for the ADF test is 0.9814 which is greater than the significant level $(0.05)$ and confirms the non-stationarity of the data. For this data, stationarity is attained with three orders of differencing ( $\mathrm{p}$-value for ADF of difference 3 is 0.0007). But the lag in the ACF plot goes into the far negative zone fairly quick for the 3rd differencing, which is an indication, the series might have been over differenced. Therefore, we deliberately select the second order of differencing although the series is not perfectly stationary (weak stationarity). This implies the ARIMA $(p, d, q)$ model will be ARIMA $(p, 2, q)$, with 2 representing the difference of order 2.

\subsection{Parameter Estimation}

Figures 4(a)-(c) show the time plot, ACF and PACF plots for the $2^{\text {nd }}$ order differencing of the GDP data. From the ACF plot, we can note that at lag 1 the ACF is significant and we can fit an MA (1) term. The first 2 lags in the PACF plot are 
also significant, the significance level shuts off suddenly after this and then surges again. Hence, to maintain a simple model, we can fit an AR (2) term. Thus, the ARIMA $(p, d, q)$ could be a candidate model where $p=2, d=2, q=1$

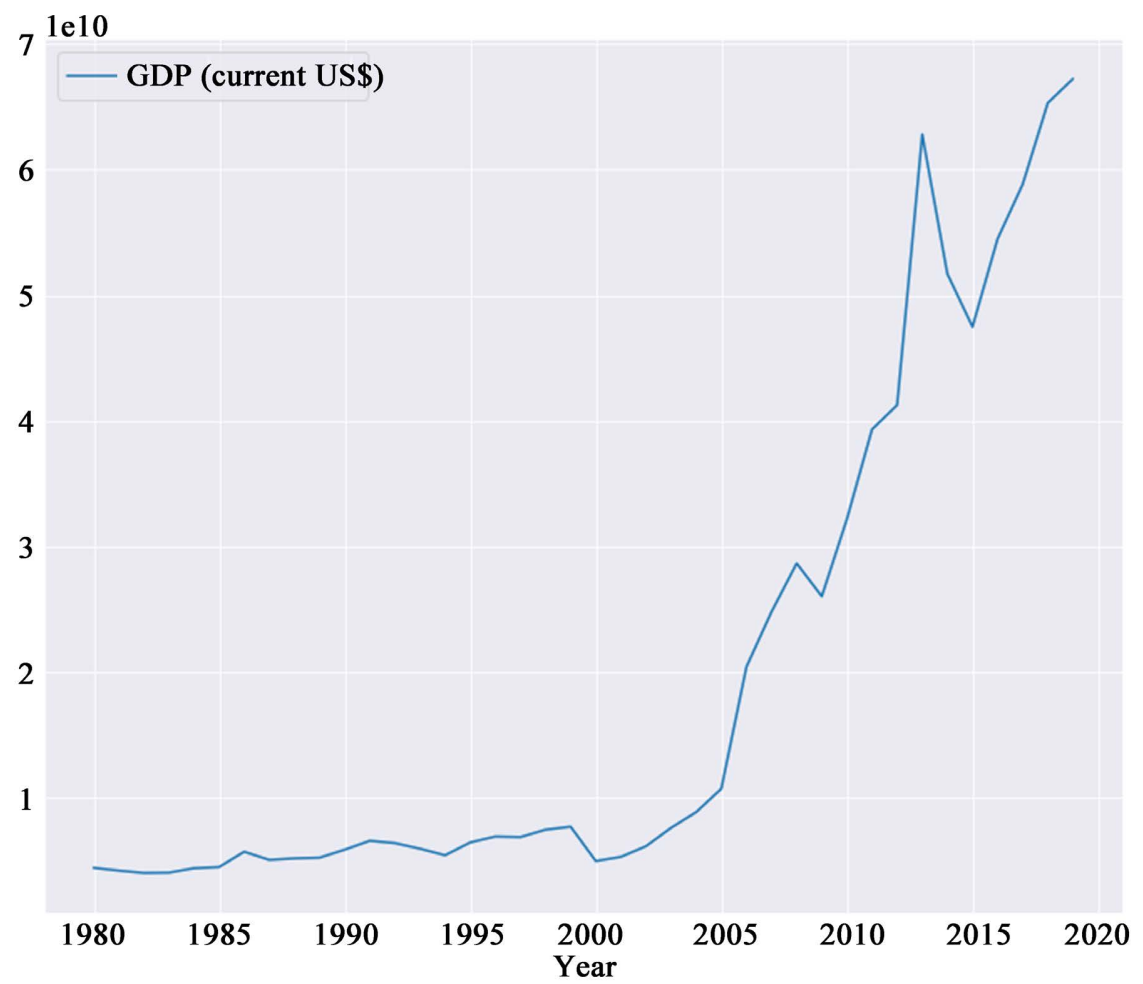

Figure 1. Time plot of GDP data.

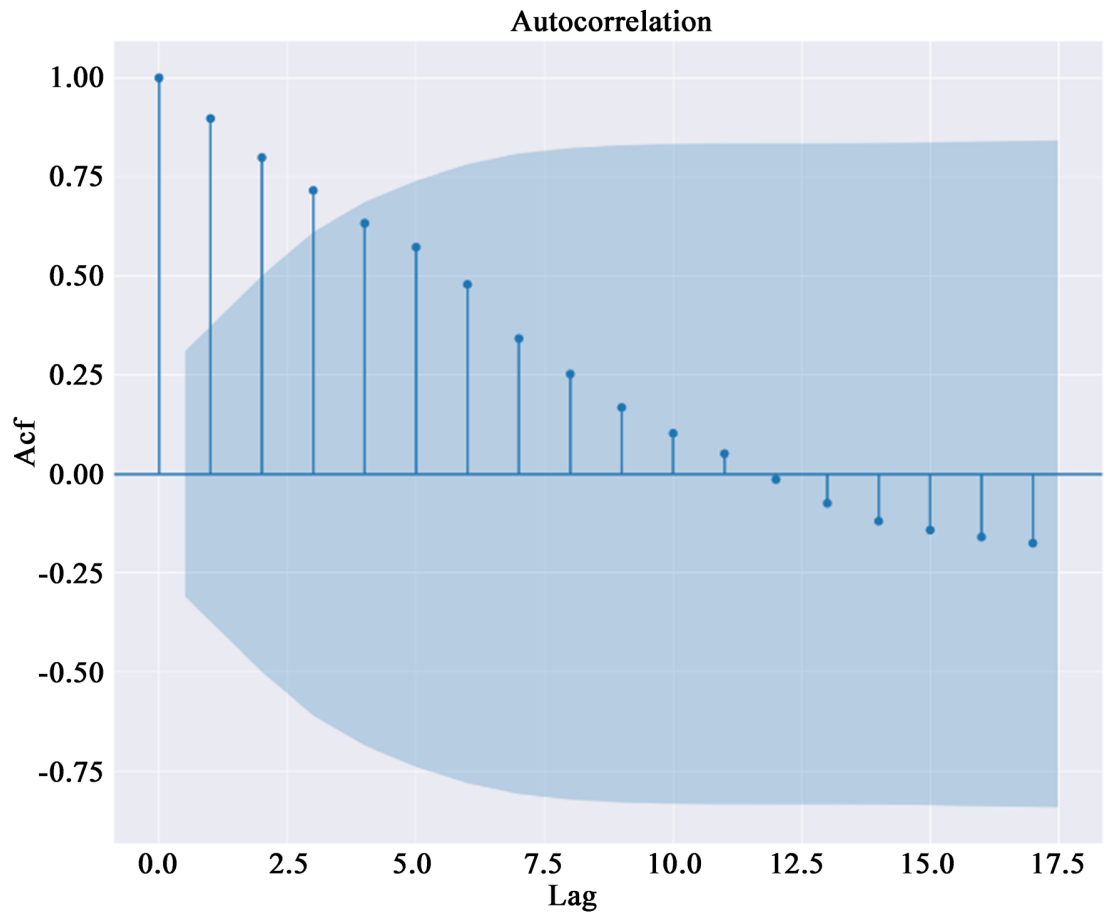

Figure 2. Acf plot of GDP data. 


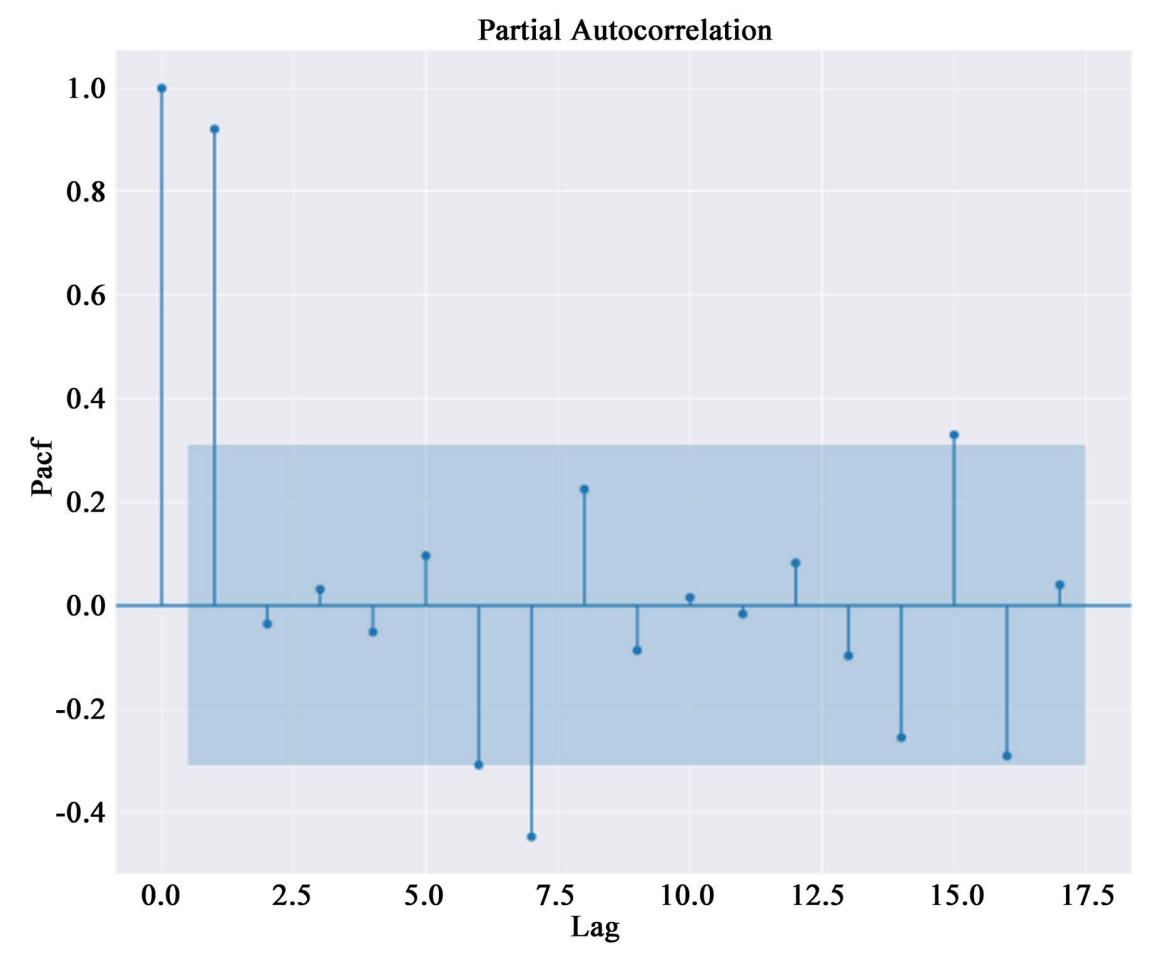

Figure 3. Pacf plot of GDP data.

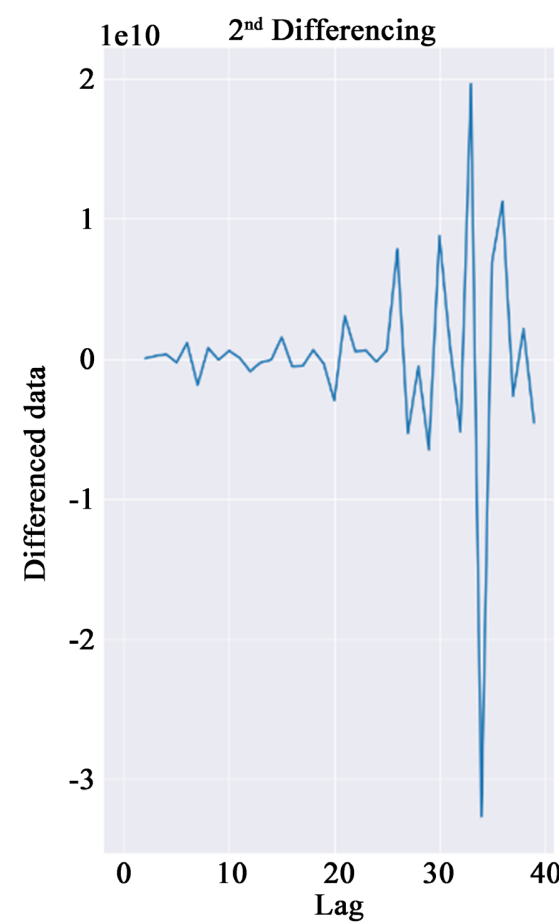

(a)

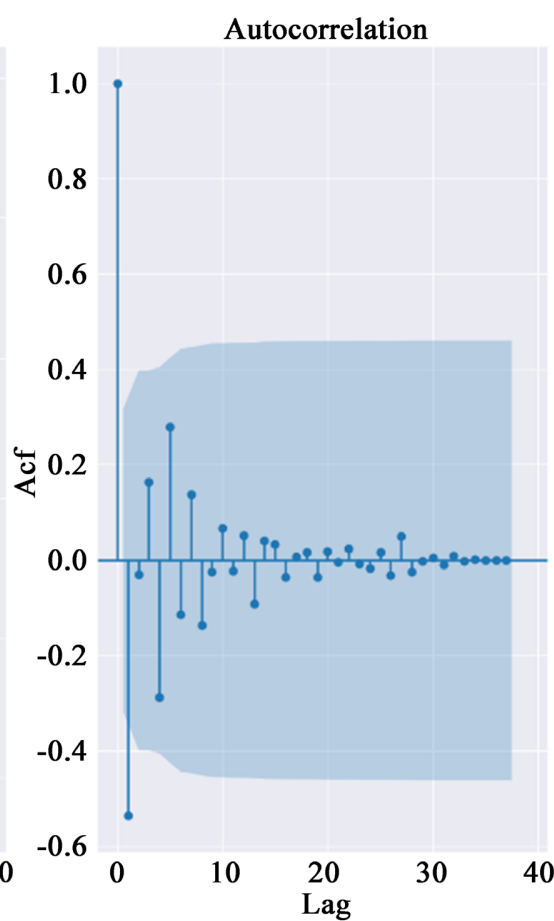

(b)

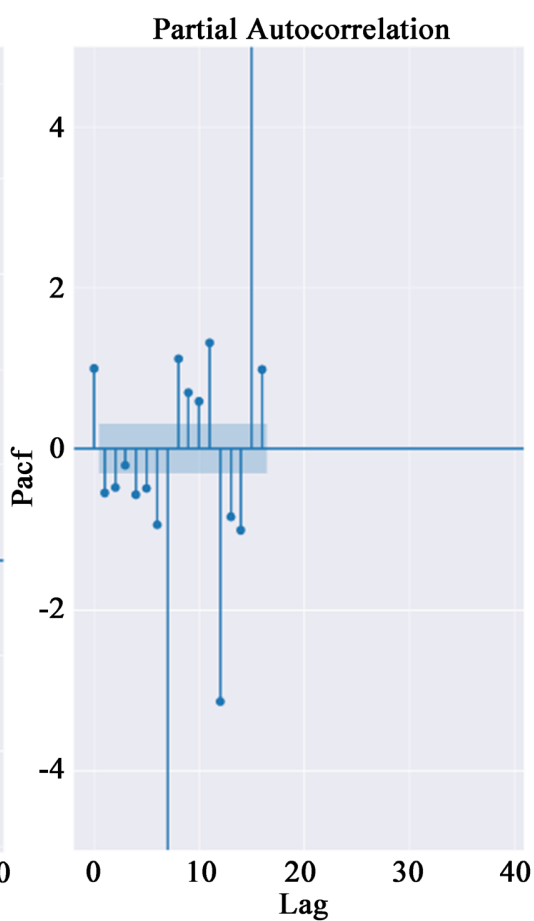

(c)

Figure 4. $2^{\text {nd }}$ order differencing of GDP data.

giving an ARIMA $(2,2,1)$ model. However, we need to select the optimal model by applying different possible ARIMA $(p, 2, q)$ models to check for the order with the least AIC. 
We implement the auto-arima function which uses a stepwise approach to automatically extract the best ARMA $(p, q)$ parameters, and by comparing AIC scores, selects the model that has the least AIC value as the best. This gave the best model as ARIMA $(2,2,2)$ with the AIC of 1807.540 from combinations of $p$ $=(0$ to 3$)$ and $q=(0$ to 3$)$. The output results of the ARIMA $(2,2,2)$ model is displayed in Table 1.

\subsection{Diagnostics Test of the Found Model}

Figures 5(a)-(e) show the residual plot of the ARIMA $(2,2,2)$ model, the histogram (density plot), the normal Q-Q plot, the Correlogram (ACF plot) and PACF plot of the residuals respectively. It can be seen from the standardized residual that the residual errors seem to alternate around a zero mean and shows non-uniform variance/heteroscedasticity. The normality assumption as seen in the histogram shows that kde line follows the $\mathrm{N}(0,1)$ line, suggesting the residuals are normally distributed. It can be seen from the normal Q-Q plot that not all the dots fall in line. The Correlogram in 5 (d) shows the residual errors are not autocorrelated, also the PACF plot shows the coefficients lying within the significance region. We can say that the error terms are independent identically distributed based on these lags. However, we use the Ljung-Box test also known as the modified Box-Pierce test to ensure our model meets the assumption that the residuals are white noise (i.i.d). The result from the test gave a p-value of 1.00 which is above 0.05 hence, we fail to not accept the null hypothesis and conclude the errors are white noise.

We can see our model is heteroscedastic, but not necessarily a bad one. Hence, we want to remove the dependence of the error variance on time so as to get a

Table 1. Output result of ARIMA $(2,2,2)$ model.

\begin{tabular}{ccccc}
\hline Dep. Variable: & \multicolumn{1}{c}{$\mathrm{y}$} & No. Observations: & $\mathbf{4 0}$ \\
\hline Model: & SARIMAX $(2,2,2)$ & Log Likelihood & -898.770 \\
AIC & 1807.540 & & BIC & 1815.727 \\
HQIC & 1810.453 & & & \\
\hline Variable & coef & std err & $\mathbf{z}$ & P-value \\
\hline ar.L1 & -1.1055 & 0.102 & -10.823 & 0.000 \\
ar.L2 & -0.3411 & 0.191 & -1.78 & 0.074 \\
ma.L1 & 0.1244 & 0.210 & 0.592 & 0.554 \\
ma.L2 & -0.8445 & 0.247 & -3.413 & 0.001 \\
sigma2 & $2.103 \mathrm{e}+19$ & $5.93 \mathrm{e}-20$ & $3.55 \mathrm{e}+38$ & 0.000 \\
Ljung-Box (Q): & 11.77 & & Jarque-Bera (JB): & 35.58 \\
Prob (Q): & 1.00 & & Prob (JB): & 0.00 \\
Heteroskedasticity (H): & 44.66 & & Skew: & 0.63 \\
Prob (H) (two-sided): & 0.00 & & Kurtosis: & 7.57 \\
\hline
\end{tabular}



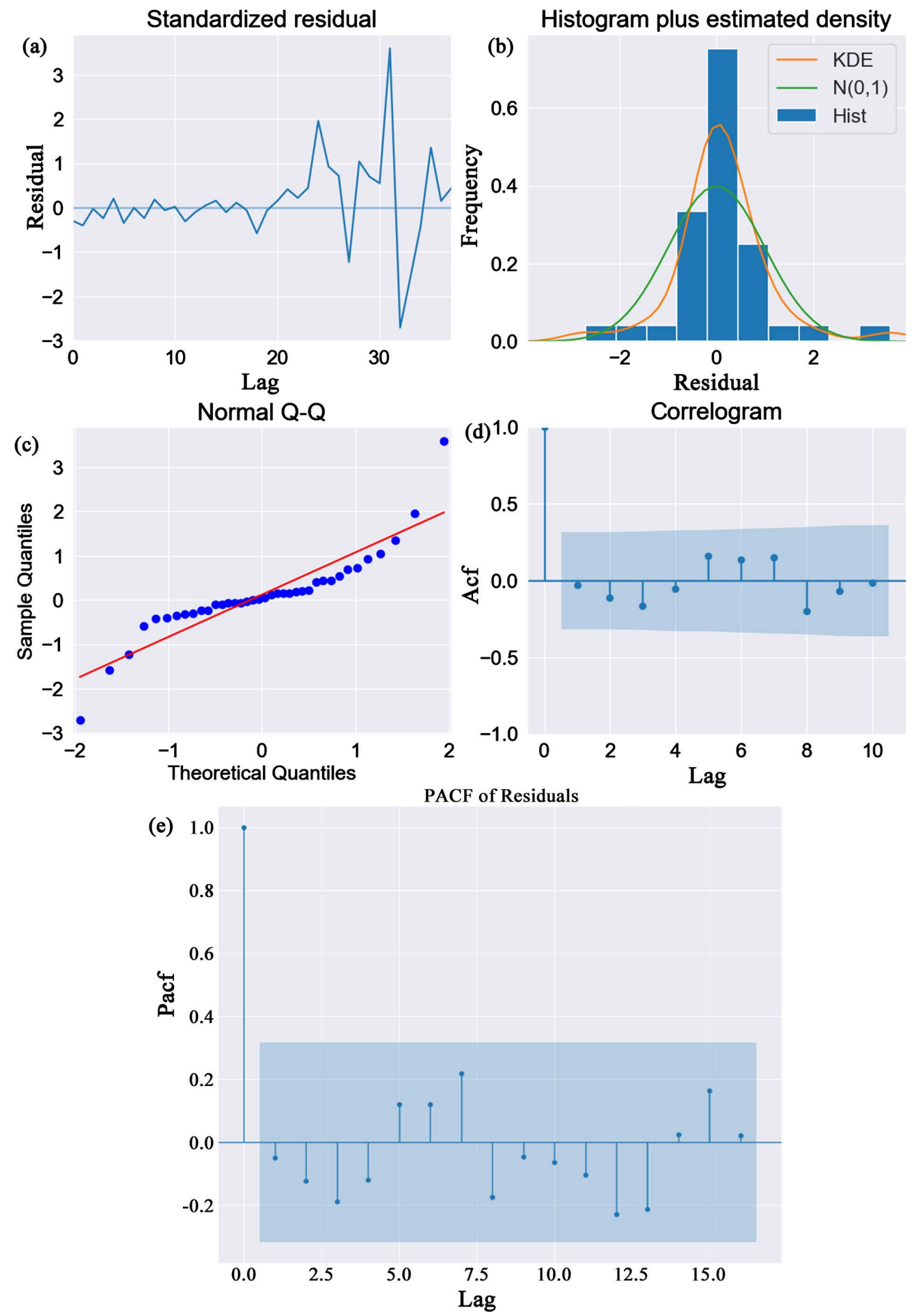

Figure 5. Diagnostics of the ARIMA $(2,2,2)$ model.

better model. The residuals of our ARIMA model were fitted with a GARCH (1, 1) model and the results are shown in Table 2. The interpretations of our GARCH model results are taken from [20]. The beta coefficient was 0.6985 which is close to 1 but a little lower. This is good because it is basically our MA term, so in terms of volatility of our model, we want beta to be less than 1 in ab- 
solute terms. A large $\beta$ value causes $\sigma(t)$ to be highly correlated with $\sigma^{2}(t-1)$ resulting in the conditional standard deviation process having a relatively long-term persistence. The coefficient of omega was $1.8361 \mathrm{e}+18$, this is the baseline variance for the model. The standard deviation of the GDP would be the square-root of omega. According to this coefficient, the standard deviation should be around 1,355,027,674.99 per year. The alpha coefficient was also 0.3015 , which indicates how much volatility from the previous period will be carried over into the next period. Adding this to our beta coefficient gives a number of exactly 1.00 , giving us a random walk model.

We expect our GDP will be about 561,920,000 US\$ per year as indicated by the mu coefficient.

\subsection{Forecasting with Our ARIMA-GARCH Model}

The final forecast equation of our ARIMA-GARCH model is given in Equation (12).

Figure 6 shows the plot of the fitted ARIMA $(2,2,2)$ model against the observed data. Figure 7 and Table 3 show the forecast values of Ghana's GDP for the next 10 years, using our ARIMA $(2,2,2)$ model. The table also includes the lower and upper values for $95 \%$ confidence interval. This shows an increase in the GDP current value of Ghana.

After fitting the GARCH $(1,1)$ model on the residuals of our ARIMA model, our final ARIMA-GARCH forecast is shown in Figure 8. Table 4 shows the ARIMA-GARCH forecast values and also the forecast values of the International Monetary Fund (IMF). Our ARIMA-GARCH model predicted the GDP for the year 2020 to be $73,856,010,000$, which can be compared to the actual GDP released by the World Bank for 2020 which was $72,354,428,865$.

Table 2. Constant Mean-GARCH Model Results.

\begin{tabular}{ll}
\hline Dep. Variable: 0 & R-squared: 0.000 \\
Mean Model: Constant Mean & Adj. R-squared: 0.000 \\
Vol Model: GARCH & Log-Likelihood: -889.036 \\
Distribution: Normal & AIC: 1786.07 \\
Method: Maximum Likelihood & BIC: 1792.62 \\
No. Observations: 38 & Df Model: 1
\end{tabular}

Df Residuals: 37

\begin{tabular}{ccccc}
\hline \multicolumn{5}{c}{ Mean Model } \\
\hline Variable & coef & std err & $\mathbf{t}$ & P-value \\
\hline mu & $5.6192 \mathrm{e}+08$ & $1.109 \mathrm{e}+09$ & 0.507 & 0.612 \\
\hline \multicolumn{5}{c}{ Volatility Model } \\
Variable & coef & std err & $\mathbf{t}$ & P-value \\
\hline omega & $1.8361 \mathrm{e}+18$ & $3.758 \mathrm{e}+18$ & 0.489 & 0.625 \\
alpha [1] & 0.3015 & 0.308 & 0.980 & 0.327 \\
beta [1] & 0.6985 & 0.483 & 1.448 & 0.148 \\
\hline
\end{tabular}




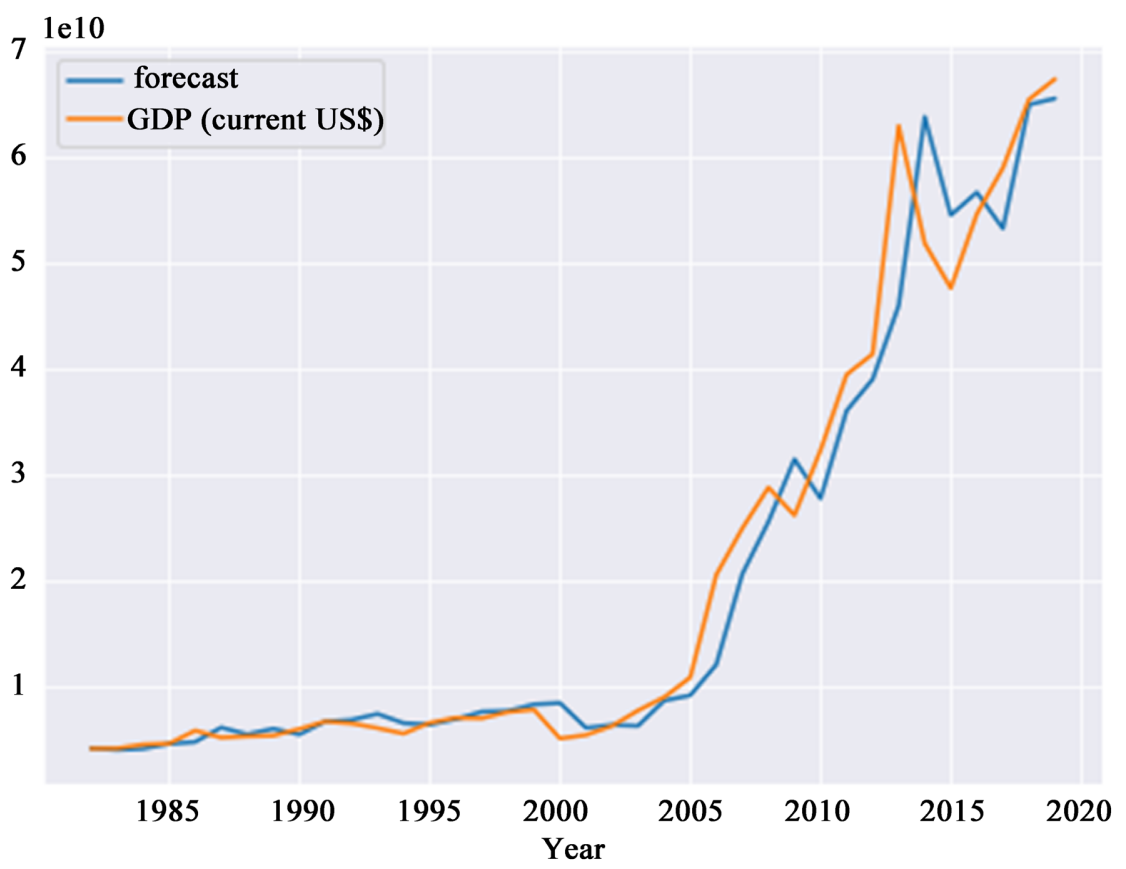

Figure 6. Fitted values against observed data.

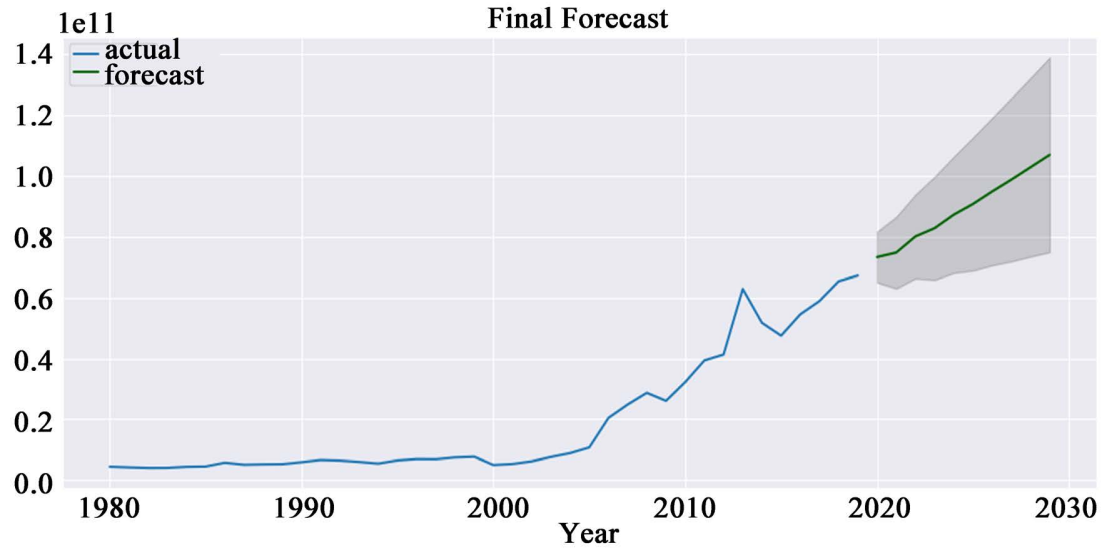

Figure 7. $\operatorname{ARIMA}(2,2,2)$ forecast.

Table 3. ARIMA forecast of Ghana's GDP for ten years.

\begin{tabular}{cccc}
\hline Year & Forecast Series & Lower Series & Upper Series \\
\hline 2020 & $73,294,100,000$ & $64,946,690,000$ & $81,641,500,000$ \\
2021 & $74,748,370,000$ & $62,973,240,000$ & $86,523,500,000$ \\
2022 & $80,049,030,000$ & $66,266,530,000$ & $93,831,520,000$ \\
2023 & $82,755,470,000$ & $65,774,570,000$ & $99,736,380,000$ \\
2024 & $87,201,750,000$ & $68,093,570,000$ & $106,309,900,000$ \\
2025 & $90,713,290,000$ & $68,844,470,000$ & $112,582,100,000$ \\
2026 & $94,825,090,000$ & $70,588,010,000$ & $119,062,200,000$ \\
2027 & $98,716,310,000$ & $71,870,330,000$ & $125,562,300,000$ \\
2028 & $102,792,200,000$ & $73,427,560,000$ & $132,156,900,000$ \\
2029 & $106,873,000,000$ & $74,898,710,000$ & $138,847,200,000$ \\
\hline
\end{tabular}




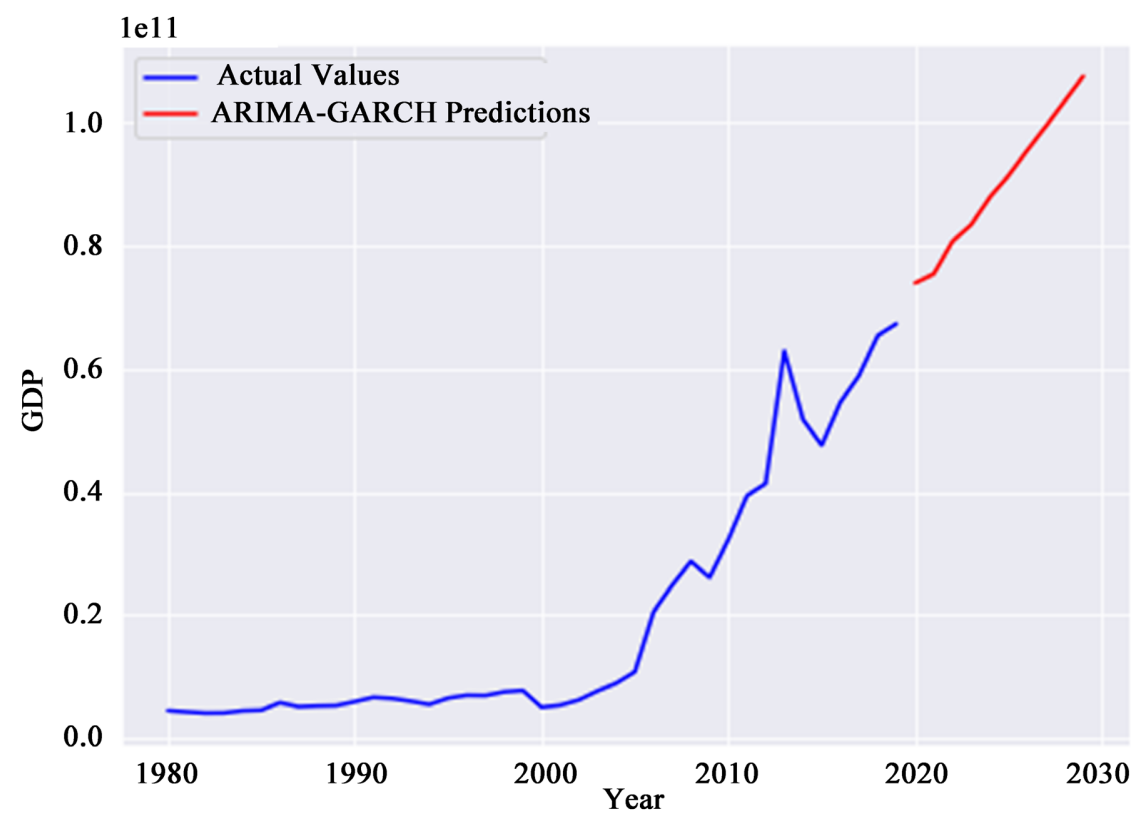

Figure 8. ARIMA-GARCH forecast.

Table 4. ARIMA-GARCH forecasts versus IMF forecasts.

\begin{tabular}{lcc}
\hline Year & Forecast & IMF Forecast \\
\hline 2017 & $61,805,770,000$ & $60,385,000,000$ \\
2018 & $67,994,920,000$ & $67,259,000,000$ \\
2019 & $72,798,710,000$ & $68,353,000,000$ \\
2020 & $73,856,010,000$ & $68,498,000,000$ \\
2021 & $75,310,280,000$ & $75,487,000,000$ \\
2022 & $80,610,940,000$ & $82,018,000,000$ \\
2023 & $83,317,390,000$ & $87,736,000,000$ \\
2024 & $87,763,660,000$ & $94,045,000,000$ \\
2025 & $91,275,200,000$ & $101,028,000,000$ \\
2026 & $95,387,010,000$ & $108,370,000,000$ \\
2027 & $99,278,220,000$ & \\
2028 & $103,354,200,000$ & \\
2029 & $107,434,900,000$ & \\
\hline & & \\
\hline
\end{tabular}

\section{Conclusions}

In this study, we implemented the ARIMA-GARCH model for forecasting the GDP of Ghana. It is observed that the residuals of our fitted ARIMA $(2,2,2)$ model show heteroscedasticity and therefore, we incorporate a GARCH $(1,1)$ model in order to capture these changes in variance over time. From this study, we can conclude that the ARIMA-GARCH model is effective in removing the error variance and improving forecasts. In terms of performance, the results 
show that the combined model outperforms the classic ARIMA model. The results of this study show that the GDP of Ghana follows a random walk and will continue to increase for the next 10 years. The model predicts that by 2029 , Ghana's GDP would be approximately 107,434,900,000 US dollars.

This introduction of non-linear time series was due to the data's bursty property; within the theory of statistics, if a time series exhibits bursty behavior, then its variance changes with time. However, its prediction methodology is not as straight forward and also determining its prediction accuracy is a challenge. Further research might investigate the efficiency and accuracy of the ARIMA-GARCH model.

Moreover, as seen in past years (years of dip), we can say there is the likelihood of the GDP of Ghana being affected by some unpredicted circumstances in the future. In general, the model's prediction result conforms to the actual situation and trend of Ghana's economic development. More information on how to deal with the effects of these events such as the covid situation and the fall in global fuel prices, would help us to establish a greater degree of accuracy on the matter. As a result, certain measures can be taken to make up for such instances, so that the economy will not be too affected causing major dips which may in turn affect the growth rate.

\section{Conflicts of Interest}

The authors declare no conflicts of interest.

\section{References}

[1] Lepenies, P. (2016) The Power of a Single Number: A Political History of GDP. New York, Columbia University. https://doi.org/10.7312/lepe17510

[2] Worldometer (n.d.) GDP. https://www.worldometers.info/gdp/what-is-gdp/

[3] Todaro, M.P. and Smith, S. (2012) Economic Development. 11th Edition, Pearson Education Inc., Boston, 37-73.

[4] Smith, L. (2021, June 30) Does High GDP Mean Economic Prosperity? https://www.investopedia.com/articles/economics/08/genuine-progress-indicator-g pi.asp

[5] Silja, F. (2019) IMF World Economic Outlook Puts Ghana in the Lead. https://www.dw.com/en/imf-world-economic-outlook-puts-ghana-in-the-lead/a-48 $\underline{356052}$

[6] Tasneem, J.D. (2021) The Fastest Growing Economy in Africa: How Ghana Got It Right.

https://www.gvi.co.uk/blog/the-fastest-growing-economy-in-africa-how-ghana-gotit-right/

[7] Salinas, D., Flunkert, V., Gasthaus, J. and Januschowski, T. (2020) DeepAR: Probabilistic Forecasting with Autoregressive Recurrent Networks. International Journal of Forecasting, 36, 1181-1191.

[8] Box, G.E.P. and Jenkins, G.M. (1968) Some Recent Advances in Forecasting and Control. Journal of the Royal Statistical Society, Series C (Applied Statistics), 17, 91-109. https://doi.org/10.2307/2985674 
[9] Asenso, T.Q., Erasmus, T.-B. and Mbele, B.M. (2017) Time Series Modeling and Forecasting GDP in the Ghanaian Economy. IISTE Mathematical Theory and Modeling, 7, 63-70.

[10] Zhou, B., He, D. and Sun, Z. (2014) Traffic Modeling and Prediction Using ARIMA/GARCH Model. Centre for Communication System and Research, University of Surrey, Guildford, $13 \mathrm{p}$.

[11] Higgins, P., Zha, T. and Zhong, K. (2016) Forecasting China's Economic Growth and Inflation. Working Paper No. 22402, National Bureau of Economic Research, Cambridge. https://doi.org/10.3386/w22402

[12] Tran, Q., Ma, Z., Li, H., Hao, L. and Trinh, Q. (2015) A Multiplicative Seasonal ARIMA/GARCH Model in EVN Traffic Prediction. International Journal of Communications, Network and System Sciences, 8, 43-49.

https://doi.org/10.4236/ijcns.2015.84005

[13] Eersel, S. (2019) Forecasting African GDP Growths Using Factor Models and Machine Learning Methods. Published Bachelor Thesis, Erasmus University Rotterdam, Rotterdam, $30 \mathrm{p}$.

[14] Dierckx, T. (2020, September 10) ARIMA-GARCH Forecasting with Python. https://medium.com/analytics-vidhya/arima-garch-forecasting-with-python-7a3f79 $\underline{7 \mathrm{de} 3 \mathrm{ff}}$

[15] Box, G.E.P., Jenkins, G.M., Reinsel, G.C. and Ljung, G.M. (2016) Time Series Analysis, Forecasting and Control. 5th Edition, John Wiley \& Sons. Inc., Hoboken, 54-68.

[16] Ranjith, K.K. (2020, January 15) Time Series Model(s)-ARCH and GARCH. https://medium.com/@ranjithkumar.rocking/time-series-model-s-arch-and-garch-2 781a982b448

[17] Quantstart (n.d.) Generalized Autoregressive Conditional Heteroskedasticity $\operatorname{GARCH}(\mathrm{p}, \mathrm{q})$ Models for Time Series Analysis.

https://www.quantstart.com/articles/Generalised-Autoregressive-Conditional-Heter oskedasticity-GARCH-p-q-Models-for-Time-Series-Analysis/

[18] Bollerslev, T., Chou, R.Y. and Kroner, K.F. (1992) ARCH Modeling in Finance: A Review of the Theory and Empirical Evidence. Journal of Econometrics, 52, 5-59. https://doi.org/10.1016/0304-4076(92)90064-X

[19] Nakatsuma, T. and Tsurumi, H. (1996) ARMA-GARCH Models: Bayes Estimation Versus MLE, and Bayes Non-stationary Test. Working Papers No, 199619, Department of Economics, Retgurs University, New Jersey.

[20] Barnes Analytics (2017) GARCH Models in Python. https://barnesanalytics.com/garch-models-in-python/ 